or NSTEMI) as well as meta-regression according to starting year and mean year of study.

Results A total of 26 studies were included with over 16 million participants incorporating 3.3 million weekend and over 12.5 million weekday admissions and the rates of mortality rates were $18.3 \%$ and $15.7 \%$, respectively. The pooled results of all 26 studies suggest that weekend admission was associated with a small increase in risk of early mortality (OR 1.05 95\% CI 1.03-1.07). The results for subgroups of STEMI and NSTEMI cohorts were not statistically significant and timing of admission after 2005 had minimal influence on the results (OR 1.06 95\% CI 0.95-1.17).

Conclusions There is a small weekend effect for admission with acute coronary syndrome that has persisted over time.

\section{DO HIGH SENSITIVITY TROPONIN ASSAYS ACTUALLY RESULT IN A SHORTER HOSPITAL STAY IN A REAL WORLD CLINICAL SETTING?}

${ }^{1}$ Arvind Singhal ${ }^{*}$, ${ }^{1}$ lain Parsons, ${ }^{2}$ Ed Nicol. ${ }^{1}$ Chelsea and Westminster Hospital; ${ }^{2}$ Royal Brompton Hospital

\subsection{6/heartjnl-2017-311726.68}

Background Hospitals face an immense bed pressure this winter. Admission to hospital for rule-out of MI is common, and early rule-out may expedite patient turnover.

Aims To assess the time to discharge following introduction of high sensitivity troponin I (hsTnI) in comparison to standard Troponin assays.

Methods A prospective cohort study was undertaken over a 3 month period following the introduction of hsTnI (ARCHITECT assay) at Chelsea and Westminster Hospital in London. Troponin assays were taken at presentation and after 3 hours. A positive result was defined as a high baseline troponin (>68 ng/L for males, $>30 \mathrm{ng} / \mathrm{L}$ for females) or a change of $>50 \%$ between the two samples with at least one sample above the 99th centile for gender. The assay results were matched to patient demographics, admission and discharge times and discharge diagnoses. All troponin testing other than that performed at admission were excluded. Results were compared against the conventional Troponin I assay (threshold of detection $32 \mathrm{ng} / \mathrm{L}$ ), with the 12 hour rule-out protocol, using 6 months of data prior to the introduction of the hsTnI assay. Results hsTnI were requested on 553 patients in the emergency department over a 3 month period (6.1 patients/day), compared with 647 patients who had conventional troponin I testing over a preceding 6 month period (3.6 patients/day).

The mean length of hospital stay was 3.83 days in the hsTnI group vs 2.92 days in the conventional troponin group $(p=0.04)$. The median length of stay was 0.82 days in both groups $(p=0.09)$. All patients who had 2 hsTnI requests ended up being admitted to hospital, even if both were undetectable. No patient who had an undetectable 1st troponin had a positive 2nd troponin. Overall 93/553 (17\%) hsTnI results were identified as positive, of which 33/553 (6\%) had acute coronary syndromes, with 22/553 (4\%) undergoing coronary intervention. 19/553 (3\%) had positive hsTnI from other cardiac causes 41/553 (7\%) had non-cardiac causes.

Conclusions The introduction of hsTnI has not resulted in a shorter length of hospital stay for patients undergoing troponin testing. Troponin testing became more frequent after the introduction of the new assay which may have resulted in extra, unnecessary admissions. Acute coronary syndromes were relatively uncommon. Combining early rule-out algorithms, such as discharging patients whose initial hsTnI measurement is undetectable, and more judicious use of troponin testing, may prevent unnecessary admissions.

Conflict of interests Nil

\section{CLINICAL OUTCOMES OF CLOPIDOGREL VERSUS TICAGRELOR IN THE ELDERLY PATIENTS WITH ACUTE CORONARY SYNDROME}

Lampson Fan, Asad Shabbir*, Stewart Mclure, Jocelyn Lam, Mark Cassar, Nicos Spyrou. Royal Berkshire Hospital

\subsection{6/heartjnl-2017-311726.69}

Background Acute coronary syndrome (ACS) remains one of the leading causes of death in the UK. One of the recent advances in treatment in the treatment of ACS is ticagrelor, a potent $\mathrm{P}_{2} \mathrm{Y}_{12}$ inhibitor, that has become the recommended anti-platelet by the ESC for the treatment of ACS due to its mortality benefit over clopidogrel. The increased anti-platelet inhibition however, also results in significantly increased nonCABG related bleeding. With the ageing population there is a rising proportion of elderly patient $(>75)$ presenting with ACS. The elderly patients generally have greater co-morbidities, higher risk of recurrent ischaemia and also higher risk of bleeding but there is a distinct lack of evidence for this cohort of patients. In the landmark PLATO study for example, only $15 \%$ of patients were over the age of 75 . It is unclear currently whether ticagrelor is beneficial in these elderly patients. Methods A retrospective cohort study was performed in patients aged 75 and over who were admitted to the Royal Berkshire Hospital (RBH) between 2013 and 2015 with acute coronary syndrome. Patients were discharged either on clopidogrel (147 patients) or ticagrelor (154 patients) according to the local trust protocol at the time. Clinical characteristics and 12 month clinical outcomes (all-cause mortality, cardiovascular deaths, bleeding, angina, STEMI, NSTEMI, dyspnoea and ischaemic stroke) were analysed. Crusade score was used to calculate the bleeding risk.

Results The age and baseline clinical characteristics are similar between the two groups. Both had moderate bleeding risk with mean Crusade score of 36.9 for the clopidogrel group and 37 for the ticagrelor group. There was no significant difference in all-cause mortality $(8 \%$ vs $10 \%, \mathrm{p}=0.55)$, cardiovascular mortality $(2.7 \%$ vs $1.9 \%, \mathrm{p}=0.72)$, ischaemic stroke $(0.6 \%$ vs $1.9 \%, p=0.62)$, admissions with non-cardiac chest pain $(5.2 \%$ vs $6.1 \%, \mathrm{p}=0.8)$, angina $(6.1 \%$ vs $5.2 \%, \mathrm{p}=0.8)$ or STEMI ( $2 \%$ vs $1.3 \%, \mathrm{p}=0.67)$ between patients discharged on clopidogrel or ticagrelor. Patients on clopidogrel however had significant increased re-admissions with NSTEMI compared to ticagrelor $(7.5 \%$ vs $1.9 \%, p=0.028)$. No increase in either major ( 8.4 vs $8.1 \%, \mathrm{p}=1)$ or minor bleeding $(20 \%$ vs $17 \%, p=1$ ) was observed in the ticagrelor compared to the clopidogrel group using TIMI-bleeding criteria. Only 1 patient reported significant dyspnoea in the ticagrelor group.

Conclusions Elderly patients discharged on ticagrelor had reduced re-admissions with NSTEMI without an increase in either major or minor bleeding compared to patients discharged on clopidogrel. 